\title{
Lessons from Studies in Middle-Aged and Older Adults Living in Mediterranean Islands: The Role of Dietary Habits and Nutrition Services
}

\author{
Stefanos Tyrovolas and Evangelos Polychronopoulos \\ Department of Nutrition and Dietetics, Harokopio University, 17671 Kallithea, El. Benizelou 70, Attica, Athens, Greece
}

Correspondence should be addressed to Evangelos Polychronopoulos, epolychronopoulos@yahoo.com

Received 31 August 2010; Accepted 9 September 2010

Academic Editor: Demosthenes Panagiotakos

Copyright (C) 2011 S. Tyrovolas and E. Polychronopoulos. This is an open access article distributed under the Creative Commons Attribution License, which permits unrestricted use, distribution, and reproduction in any medium, provided the original work is properly cited.

Background. Islands in the Mediterranean basin share particular habits and traditions and greater life expectancy than other European regions. In this paper, particular interest has been given to the effect of the Mediterranean diet, as well as nutritional services on CVD risk, on Mediterranean islands. Methods. Published results from observational studies were retrieved from electronic databases (Pubmed and Scopus) and summarized. Results. Prevalence of CVD risk factors is increased. Adherence to the Mediterranean diet was moderate, even among the elderly participants. Furthermore, the presence of a dietician was associated with higher adherence to the Mediterranean dietary pattern and consequently lowers CVD risk. Conclusion. Adherence to the Mediterranean diet is reduced, while the prevalence of CVD risk factors is increasing at alarming rates. Public health nutrition policy has the opportunity to improve the health and quality of life of people living in isolated insular areas of the Mediterranean basin.

\section{Introduction}

According to the World Health Organization (WHO), the proportion of people over the age of 60 is growing faster than any other age group [1]. In many countries, the older population, over 80 years of age, is the fastest growing portion of the population [2]. Due to these changes, the global population pyramid (Figure 1) is undertaking a shift-from pyramid to cube-as the proportion of young adults' declines and the proportion of older people increases [3]. Prevention programs to reduce chronic disease risks have traditionally focused on younger adults. However, a variety of researchers have started to focus their attention on elders. This change in outlook has come about since people are now living longer, and consequently the older population is rapidly increasing and medical expenditure is rising with the onset of several major chronic diseases [4].

The role of healthy diet in the prevention and control of morbidity and premature mortality due to noncommunicable diseases, like CVD and cancer, has well been established by the vast population-based epidemiological research carried out during the last two-three decades [5, 6]. Older adults represent a group of population with special needs. Many of them lose their interest in food have chewing difficulties, difficulties in meal preparation, and more financial restraints [7]. A lot of them can show deficiencies in macromicronutrients, which may be the main cause for many cardiovascular diseases [8]. For those reasons, a Modified Pyramid for the older adults has been designed [8], that recommend to the elders emphasizing in whole grains, vegetables and fruits, also, in low-fat and nonfat forms of dairy products, including reduced lactose while they have to avoid high saturated and trans fatty acid types of oils and high saturated fat animal.

Improving diet and nutrition represent key public health targets. Mediterranean diet is a healthy dietary pattern which refers to specific food consumption patterns typical of some Mediterranean regions in the early 1960s, such as Crete, other parts of Greece, Spain, south France, and south Italy [9]. The Mediterranean dietary pattern emphasizes a consumption of fat primarily from foods high in monounsaturated fatty acids, and mainly olive oil, and encourages 


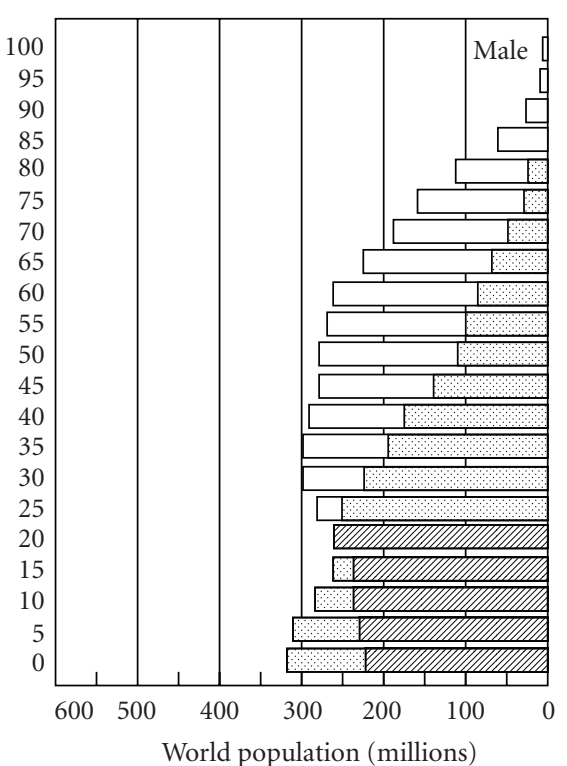

1995

2050, low fertility and low mortality 2050 , population $\leq 1995$ population

(a)

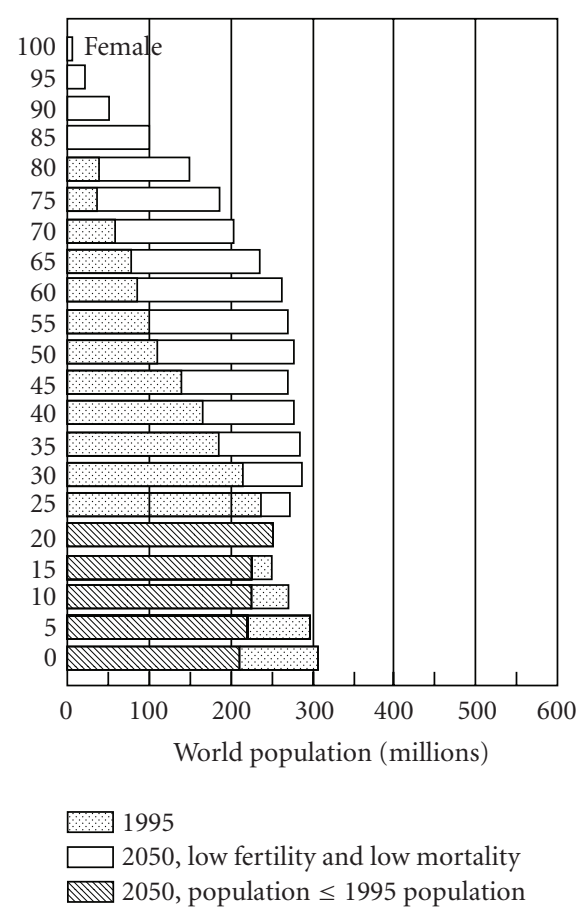

(b)

Figure 1: Global population pyramid (adopted from International Institute for Applied Systems Analysis)

the consumption of fruits, vegetables, tree nuts, legumes, whole grains, fish and poultry in low to moderate amounts, a relatively low consumption of red meat, as well as a moderate consumption of alcohol normally with meals, but the proportions of macronutrients may vary. This traditional dietary pattern, however, is not a homogeneous nutritional model. Instead, food and nutritional aspects vary between Mediterranean regions [10]. Adherence to the Mediterranean pattern has been associated with lower risk of cancer and CVD and consequently higher life expectancy $[9,11,12]$. Unfortunately, observational studies have reported strong evidence that this traditional dietary pattern, has changed to a more "westernized" type of diet in nowadays, at all ages [13-15]. This change was mainly attributed due to the rapid urbanization, economic development, and other related factors [13].

All the previously mentioned underline the importance of targeted public health services on prevention. Global planning on nutrition policies has provided by the 1992 world declaration on nutrition and plan of action on nutrition. Most countries around the world are still facing nutritionrelated problems [16]. However, the role of nutritional services on population's health status has not been well evaluated and documented, yet. Public health nutrition, even in the elderly should emphasize on planning special programs that provide food assistance, nutrition screening and education, nutrition therapy, and care management [17]. Furthermore, according to international dietetic organisations, the promotion of a healthy aging requires rectifies in the lack of nutritional services and increment in the nutritional capacity such as adequate number of staff and infrastructure [17]. Furthermore, nutritional education could be, from the public health practitioners, the way to improve health and quality of life of middle-aged and older populations [18].

This paper focuses on studies that investigated food habits on CVD risk factors, in middle-aged, and elderly populations living at the Mediterranean islands. The Mediterranean islands were selected because in a manner of speaking are more isolated than other continental areas. Moreover people living in these islands, have various habits that are quite different from those of people living on the mainland. Furthermore, the Mediterranean diet, which has been the basic nutritional model in these insular areas for many years and has been associated with lower risks of cancer and CVD mortality [9] and higher life expectancy [6].

\section{Methodology}

Table 1 summarizes the studies that will be discussed in this paper. Original research studies published in English between 1975 and 2009 were selected through a computerassisted literature search (i.e., Pubmed http://www.ihm.nlm .nih.gov/ and Scopus http://www.scopus.com/). Computer searches used combinations of keywords related to the cardiovascular disease, middle-aged and elderly populations, islands of the Mediterranean area (middle-aged population, elderly population, cardiovascular disease, coronary heart disease, cardiovascular risk factors, Mediterranean islands) and diet, nutrition or lifestyle habits. Additionally, the reference lists of the retrieved articles, which assisted in 
finding relevant-to-the-present articles that did not allocate through the searching procedure. The following information was abstracted according to a fixed protocol: design of study (cross-sectional or prospective cohort), sample size, mean age and sex of participants, followup duration, assay methods, and degree of adjustment for potential confounders. Thus, 7 studies were selected and discussed out of which 2 were prospective and 5 were cross-sectional.

\section{A Summary of the Main Findings}

3.1. The Seven Countries Study. This was the first study that included Mediterranean islands and investigated among other factors, the relationship between eating habits and long-term incidence and mortality ( 25 year followup) from cancer, CHD, and stroke in different populations $[19,20]$. Large variations, about a 10 -fold difference, in age-adjusted 25-year death rates were reported between cohorts [19]. Death rates varied greatly from east Finland exhibiting 268 per 1000, compared to 25 per 1000 observed in Crete [21]. The investigators attributed the differences in mortality rates between the 16 cohorts to the nutritional habits of the participants and, in particular, to the intake of saturated fatty acids and flavonoids. The effects of saturated and unsaturated fats on CHD mortality surfaced subsequent to this study. Dairy fat (saturated fat) was consumed in Northern Europe, whereas, in Southern Europe (the lowest risk area) fat was predominated by olive oil (unsaturated fat). Furthermore, Menotti and colleagues [20] examined the association of simple foodgroups and their combinations with 25-year mortality from CHD. Similar findings were discovered. Overall, combined vegetable foods (excluding alcohol) were inversely correlated with CHD death rates, whereas combined animal foods were directly correlated. Greek islands and Japan experienced the fewest death rates caused by CHD. These findings suggest that healthy dietary patterns are an important determinant of CHD risk, and reveal the Mediterranean diet has a major effect in the protection against CVDs.

3.2. The Mediterranean Islands Study (MEDIS). The MEDIS (MEDiterranean ISlands) study is a health and nutrition survey which aimed to evaluate bioclinical, lifestyle, behavioral and dietary characteristics of elderly people living in Greek Mediterranean islands and Cyprus Republic. According to the MEDIS study, the level of adherence to the Mediterranean diet was $61 \%$ in both men and women. Level of adherence to the Mediterranean diet (i.e., at what percent individuals' adopted the Mediterranean diet pyramid) was 55\% in Aegean islands and Cyprus Republic, 58\% in Crete and $60 \%$ in the Ionian Islands $(\mathrm{p}<0.001)$. Especially, level of adherence to the Mediterranean diet was $61 \%$ in Samothrace, $54 \%$ in Lesvos, $64 \%$ in Lemnos, 64\% Zakynthos, 59\% in Corfu and Cephalonia, 58\% in Crete, and 55\% in Cyprus Republic. The level of adherence to the traditional Mediterranean diet was moderate and people living in rural areas seem to hold onto traditional dietary habits in a better way [22]. Moreover, the researchers noticed that a healthy diet, close to the Mediterranean model, high in carbohydrates and vegetable protein is associated with a lower likelihood of being obese and may help better elderly people to preserve a normal weight [23]. Furthermore, higher adherence to the Mediterranean diet seems to be associated with lower likelihood of being an elder obese and having diabetes [24]. Also, this study evaluated the association between the consumption of various patterns and the prevalence of CVD risk factors among elderly participants. Healthier patterns, like the Mediterranean, were correlated with lower likelihood of having an elder a CVD risk factor [25].

Furthermore, the MEDIS study, revealed the association between long-term fish intake (a basic component of the Mediterranean diet) and health status [26]. Particularly, fish intake was inversely associated with systolic blood pressure $(P=0.026)$, fasting glucose $(\mathrm{p}<0.001)$, total serum cholesterol $(P=0.012)$, and triglyceride levels $(P=0.024)$. Additionally, multinomial logistic regression revealed that a reduction of $100 \mathrm{~g}$ per week in fish intake was associated with a 19\% higher likelihood of having one additional cardiovascular risk factor such as hypertension, hypercholesterolemia, diabetes, and obesity. The MEDIS study also evaluated whether alcohol consumption is associated to blood pressure [27]. The major finding was that when adjusting for confounding factors (age, sex, years of school, physical activity level, body mass index, medication use, dietary and smoking habits) a J-shaped association of alcohol intake with systolic $(P=0.001)$, diastolic $(P=0.02)$, mean $(P=0.001)$, and pulse pressure $(P=0.07)$ was observed. Therefore, longterm moderate alcohol consumption in elders may improve blood pressure levels and thus, CVD prognosis. Moreover, recently published findings from this epidemiological study revealed a positive association between coffee consumption and Body Mass Index (BMI). Specifically, coffee drinking was positively related to BMI levels, after adjustment for various confounding factors. However, when the analysis was performed separately for active and inactive participants, it has been found that only in those who were inactive, coffee drinking was significantly associated with BMI [28]. Furthermore, the MEDIS study, reported the prevalence of hypertension, hypercholesterolemia, diabetes and obesity, depressive symptomatology, smoking, and physical inactivity in the elderly; by the exception of smoking, all other rates were high, suggesting increased cardio-metabolic risk in these people [29].

Recent data related to nutritional services on the islands studied in MEDIS [30], revealed deficits on dieticians'/ nutritionists' supply in the public and private sector on the studied islands. Furthermore, in some areas there was no dietician to offer nutritional services and there were no legislated job positions for dieticians (in hospital or health centres) or no one covered the job positions. It seems that the dietician's job positions (in public health services), in these insular areas, presented under availability [30]. Finally, further analysis after various adjustments made revealed that the longer the presence of a dietician on an island the greater the level of adherence to the "healthy" Mediterranean dietary pattern of the older population (i.e., higher MedDietScore) $(P=0.03)[31]$. 
TABLE 1: A summary of studies that evaluated the role of diet on CVD risk in populations living on Mediterranean islands.

\begin{tabular}{|c|c|c|}
\hline Study & Design & Sample \\
\hline $\begin{array}{l}\text { The Seven } \\
\text { Countries } \\
\text { Study } \\
{[17-19] .}\end{array}$ & Cohort. & $\begin{array}{l}\text { Of the total Study's sample, } 1,200 \\
\text { middle-aged men ( } 40-59 \text { years old) were } \\
\text { living in Crete and Corfu islands. }\end{array}$ \\
\hline $\begin{array}{l}\text { MEDIS Study } \\
{[20-29] .}\end{array}$ & $\begin{array}{l}\text { Cross- } \\
\text { sectional. }\end{array}$ & $\begin{array}{l}553 \text { men and } 637 \text { women ( }>65 \text { years) } \\
\text { living in Cyprus republic, and in } 7 \text { Greek } \\
\text { islands (Mitilini, Samothraki, } \\
\text { Cephalonia, Crete, Corfu, Lemnos and } \\
\text { Zakynthos). }\end{array}$ \\
\hline
\end{tabular}

'Ventimiglia di Sicilia' project Prospective. [30-33]. 'Spili' study [30].

Crosssectional. 445 males and females (15-79 years) living in rural area of Crete island.

$\begin{array}{ll}\text { "Messara" } & \text { Cross- } \\ \text { study }[31] . & \text { sectional. }\end{array}$
502 (15-79 years) male farmers living in rural area of Crete island.

363 males and 472 females ( $20-70$ years) living in Sicilia island.

Crosssectional. Islands study $[34,35]$.

"Sardinia and Malta Islands" study [36].
Crosssectional.
30 mother-daughter pairs were interviewed in Sardinia and Malta islands.
Main findings

Saturated fat associated with increased CHD mortality rates. Mediterranean diet appeared a cardio-protective effect.

The level of adherence to the traditional Mediterranean diet was moderate in the elderly inhabitants of these insular areas. Adherence to this dietary pattern was associated with lower likelihood of diabetes and obesity, as well as overall CVD risk.

Lower CVD mortality in persons of ages 40 to 64 years old. Increase of total and complex carbohydrates intake and significant decrease of the consumption of total, saturated and polyunsaturated fats with increment of age.

Appeared high prevalence in many CVD risk factors. However there were no signs of postmyocardial infarction in men aged 63 and under.

The level of adherence to the traditional Mediterranean diet was moderate in the inhabitants of these insular areas. The lack of adherence to this traditional dietary pattern have led to the fact that the rural inhabitants from Crete are likely to be at a higher risk for developing CVD in comparison with earlier generations.

The elders seem to better hold the traditional Balearic Mediterranean dietary habits, than younger ones. However, Mediterranean diet appeared to being lost in the Balearic Islands, mainly in the younger generations.

The Sardo-Mediterranean dietary model is evolving under the impact of modernization - globalisation, but it is not disappearing. In Malta, the western modernity has led to a more sudden shift where the local nutrition identity is no longer Mediterranean.
3.3. The "Spili" Study and the "Messara" Study at the Greek Island of Crete. The "Spili Study" has taken place in the rural village of Spili at the island of Crete. The aim of this study was to survey the cardiovascular risk profile of a defined 'lowrisk' population [32]. The studied population comprised of men and women aged 15-79 years in the village of Spili ( $n=445)$. In this cross-sectional study the researchers found a high (44\%) prevalence of smoking in men aged 45-64 years as well as a high alcohol intake (48\% drank greater than or equal to $210 \mathrm{~g}$ of pure alcohol every week). Also, there was a high cholesterol level and a high prevalence of hypertension and diabetes. Furthermore, the investigators did not find any signs of postmyocardial infarction in Spili men aged 63 and under. According to the researchers this effect could be due to positive factors such as the closely knit social networks, the low unemployment rate, the hard water, and some of the dietary habits, for example the high consumption of olive oil, that may counter-balance the negative factors mentioned above. They also support that the low-risk factors in the past in the area of Crete could explain the low incidence of myocardial infarction today, and that this will change in the years to come.

The "Messara Study" has taken place in the rural valley of Messara on Crete Island [33]. The aim was to evaluate the changes in CVD risk factors among farmers in Crete and compare the findings with data from the 1960s (Crete islandSeven countries study). Study's population was comprised of 502 male farmers aged 15-79 years from the rural valley of Messara. According to the study, the male farmers were found to have a $30 \%$ higher BMI, a 16\% higher total 
cholesterol level, and also a not so favourable daily dietary intake compared with the Cretan farmers 45 years ago. The main finding of this study was the population's lack of adherence to the Mediterranean diet, which has led to the fact that currently farmers from Crete are likely to be at a higher risk for developing CVD compared to earlier generations.

3.4. The "Ventimiglia di Sicilia" Project. In Sicily, the greater Italic island, the "Ventimiglia di Sicilia" project began in 1989 aiming to evaluate the cardiovascular risk factors and dietary habits in a low-risk population [34]. According to this study, after the third decade of life more than $80 \%$ of this population was out of the normal weight range with a peak at 60 years and over of life for females, where the researchers found only $9.9 \%$ of subjects with a normal weight. Moreover, in this area the researchers found low mean total and LDL-cholesterol plasma concentrations and a rate of early cardiovascular mortality of 0.6 deaths/year/1000 inhabitants in subjects of ages 40-64 years in the period 1988-1997, a rate lower than that in the rest of Italy [35]. Furthermore, among the increment of age, researchers observed a significant increase of total and complex carbohydrates intake and a significant decrease of the consumption of total, saturated and polyunsaturated fats compared older with younger participants. They also observed a nonsignificant trend to decrease the daily cholesterol intake, whereas monounsaturated fat and fibre intake was not different. Calories intake strongly decreased in the people after the 6th decade of life compared with middle-aged groups [35].

3.5. The "Balearic Islands" Study. Another observational study that focused on Balearic Islands made an effort to evaluate dietary habits in the local Spanish insular population. Adherence to the Mediterranean diet among the population of the Balearic islands was found to be $43,1 \%$, and was similar in all sociodemographic and lifestyle groups, with some differences according to age, sex and physical status. The main finding was that there was an increase in the percentage of adherence with age, which was greater in males than in females. According to the researchers, the Mediterranean diet appeared to have been lost in the Balearic Islands, mainly in the younger generations [36]. Furthermore, the researchers showed from multivariate analysis that younger age groups (26-45 years) were at higher risk of being low-antioxidant consumers when compared to the $46-65$ year age group. The researchers mark that this finding appeared due to the loss of the traditional Balearic Mediterranean diet towards a more western dietary pattern [37].

3.6. The "Sardinia and Malta Islands" Study. This study was developed between 2001 and 2002 in Sardinia and Malta islands and examined dietary habits of thirty motherdaughter pairs in each insular area [38]. The design of the study allowed the researchers to show the contrast in the evolution of dietary habits over time not only between Sardinia and Malta, but also within each island. According to the study there was a trend revealing a shift away from cereals, pulses, and potatoes to the benefit of meat products, fats, and sugar. Furthermore, fruit and vegetables, olive oil, and fish, which are part of the main components of the Mediterranean diet, were among the top foods for which consumption frequency has increased in Sardinia. On the other hand, in Malta, besides an increase in olive oil and vegetable consumption, cheeses and desserts showed the highest increase. Along with modernity and improved living conditions, enhanced commercial availability and increased diversity of food preparation were also identified as factors contributing to changes in dietary habits. Finally, according to the investigators, the Sardo-Mediterranean dietary model is evolving under the impact of modernisation, but it is not disappearing. In Malta, the western modernity has led to a more sudden shift where the local nutrition identity is no longer Mediterranean but Anglo-Saxon.

\section{Conclusive Results}

The conclusion of this paper is that high adherence to a Mediterranean type of diet or a "healthy dietary" pattern was associated with reduced risk of CVD in middle-aged and even in the older people of the Mediterranean islands. Furthermore, it seems that the inhabitants of the Mediterranean islands have changed the traditional Mediterranean diet towards a more western dietary pattern. However, the elderly people living in these insular areas seem to better hold the traditional healthy dietary habits than the younger ones. The beneficial effects on blood pressure and lipids levels, body fat, and other surrogate markers of CVD risk, add biological plausibility to the epidemiologic evidence that supports a protective effect of the Mediterranean diet. Therefore, public health policy makers (physicians, dieticians, nurses, public health practitioners) should emphasize the promotion of healthy dietary patterns, like the Mediterranean diet in order to reduce the burden of CVD and improve population's quality of life. Enhancing nutritional services may contribute to holding a healthy dietary pattern and consequently improving the quality of life even in older population. Community-based, targeted nutritional policy could be one mean to this effort.

\section{References}

[1] World Health Organization. Active Ageing: A Policy Framework, http://whqlibdoc.who.int/hq/2002/who_nmh_nph_02.8 .pdf.

[2] K. Kinsella, "Demographic dimensions of global aging," Journal of Family Issues, vol. 21, no. 5, pp. 541-558, 2000.

[3] International Institute for Applied Systems Analysis (IIASA), http://www.iiasa.ac.at/Research/POP/images/image24.gif.

[4] Health, United States, With Health and Aging Chartbook, National Center for Health Statistics, Hyattsville, Md, USA, 1999.

[5] P. Kris-Etherton, R. H. Eckel, B. V. Howard, S. St. Jeor, and T. L. Bazzarre, "Lyon diet heart study: benefits of a Mediterraneanstyle, National Cholesterol Education Program/American Heart Association Step I dietary pattern on cardiovascular disease," Circulation, vol. 103, no. 13, pp. 1823-1825, 2001.

[6] A. Trichopoulou, A. Kouris-Blazos, M. L. Wahlqvist et al., "Diet and overall survival in elderly people," British Medical Journal, vol. 311, no. 7018, pp. 1457-1460, 1995. 
[7] H. Baker, "Nutrition in the elderly: diet pitfalls and nutrition advice," Geriatrics, vol. 62, no. 10, pp. 24-26, 2007.

[8] L. H. Lichtenstein, H. Rasmussen, W. W. Yu, S. R. Epstein, and R. M. Russell, "Modified MyPyramid for older adults," Journal of Nutrition, vol. 138, no. 7, p. 1400, 2008.

[9] A. Keys, A. Menotti, and M. J. Karvonen, "The diet and 15year death rate in the seven countries study," American Journal of Epidemiology, vol. 124, no. 6, pp. 903-915, 1986.

[10] A.-L. Matalas, "Disparities within traditional Mediterranean food patterns: an historical approach of the Greek diet," International Journal of Food Sciences and Nutrition, vol. 57, no. 7-8, pp. 529-536, 2006.

[11] F. Sofi, R. Abbate, G. F. Gensini, and A. Casini, "Accruing evidence about benefits of adherence to the Mediterranean diet on health: an updated systematic review and metaanalysis," The American Journal of Clinical Nutrition, vol. 92, no. 5, pp. 1189-96, 2010.

[12] M. A. Martinez-Gonzalez, M. Bes-Rastrollo, L. Serra-Majem, D. Lairon, R. Estruch, and A. Trichopoulou, "Mediterranean food pattern and the primary prevention of chronic disease: recent developments," Nutrition Reviews, vol. 67, pp. S111S116, 2009.

[13] A. Kafatos, A. Diacatou, G. Voukiklaris et al., "Heart disease risk-factor status and dietary changes in the Cretan population over the past $30 \mathrm{y}$ : the Seven Countries Study," American Journal of Clinical Nutrition, vol. 65, no. 6, pp. 1882-1886, 1997.

[14] R. Balanza, P. García-Lorda, C. Pérez-Rodrigo, J. Aranceta, M. B. Bonet, and J. Salas-Salvadó, "Trends in food availability determined by the Food and Agriculture Organization's food balance sheets in Mediterranean Europe in comparison with other European areas," Public Health Nutrition, vol. 10, no. 2, pp. 168-176, 2007.

[15] P. Marques-Vidal, P. Ravasco, C. M. Dias, and M. E. Camilo, "Trends of food intake in Portugal, 1987-1999: results from the National Health Surveys," European Journal of Clinical Nutrition, vol. 60, no. 12, pp. 1414-1422, 2006.

[16] Food and Agriculture Organization of the United Nations, "World Declaration and Plan of Action for Nutrition," FAO, Rome, Italy, 1992, http://whqlibdoc.who.int/hq/1992/ a34303.pdf.

[17] B. J. Kamp, N. S. Wellman, and C. Russell, "Position of the American dietetic association, American society for nutrition, and society for nutrition education: food and nutrition programs for community-residing older adults," Journal of Nutrition Education and Behavior, vol. 42, no. 2, pp. 72-82, 2010.

[18] N. S. Wellman, L. Y. Rosenzweig, and J. L. Lloyd, "Thirty years of the older Americans nutrition program," Journal of the American Dietetic Association, vol. 102, no. 3, pp. 348-350, 2002.

[19] W. C. Willett, "The Mediterranean diet: science and practice," Public Health Nutrition, vol. 9, no. 1 A, pp. 105-110, 2006.

[20] A. Menotti, D. Kromhout, H. Blackburn, F. Fidanza, R. Buzina, and A. Nissinen, "Food intake patterns and 25-year mortality from coronary heart disease: cross-cultural correlations in the Seven Countries Study," European Journal of Epidemiology, vol. 15, no. 6, pp. 507-515, 1999.

[21] "Lessons from the seven countries study," in CVD Risk Factor and Trends in Greece, H. Toshima, Y. Koga, and H. Blackburn, Eds.A Dontas, Ed., Springer, Tokyo, Japan, 1994.

[22] S. Tyrovolas, E. Polychronopoulos, V. Bountziouka et al., "Level of adherence to the mediterranean diet among elderly individuals living in mediterranean Islands: nutritional report from the medis study," Ecology of Food and Nutrition, vol. 48, no. 1, pp. 76-87, 2009.

[23] S. Tyrovolas, T. Psaltopoulou, and G. Pounis, "Nutrient intake in relation to central and overall obesity status among elderly people living in the Mediterranean islands: the MEDIS study," Nutrition, Metabolism \& Cardiovascular Diseases. In press.

[24] S. Tyrovolas, V. Bountziouka, N. Papairakleous et al., "Adherence to the Mediterranean diet is associated with lower prevalence of obesity among elderly people living in Mediterranean islands: the MEDIS study," International Journal of Food Sciences and Nutrition, vol. 60, supplement 6, pp. 137150, 2009.

[25] D. Panagiotakos, V. Bountziouka, A. Zeimbekis, I. Vlachou, and E. Polychronopoulos, "Food pattern analysis and prevalence of cardiovascular disease risk factors among elderly people from Mediterranean islands," Journal of Medicinal Food, vol. 10, no. 4, pp. 615-621, 2007.

[26] D. B. Panagiotakos, A. Zeimbekis, V. Boutziouka et al., "Longterm fish intake is associated with better lipid profile, arterial blood pressure, and blood glucose levels in elderly people from Mediterranean islands (MEDIS epidemiological study)," Medical Science Monitor, vol. 13, no. 7, pp. CR307-CR312, 2007.

[27] D. B. Panagiotakos, G. Kourlaba, A. Zeimbekis, P. Toutouzas, and E. Polychronopoulos, "The J-shape association of alcohol consumption on blood pressure levels, in elderly people from Mediterranean Islands (MEDIS epidemiological study)," Journal of Human Hypertension, vol. 21, no. 7, pp. 585-587, 2007.

[28] M. Yannakoulia, S. Tyrovolas, V. Bountziouka et al., "The mediating effect of physical activity and smoking on the relationship between coffee drinking and body weight in elderly individuals: the mediterranean islands study," Journal of the American Geriatrics Society, vol. 58, no. 6, pp. 12081210, 2010.

[29] S. Tyrovolas, C. Lionis, A. Zeimbekis et al., "Increased body mass and depressive symptomatology are associated with hypercholesterolemia, among elderly individuals; Results from the MEDIS study," Lipids in Health and Disease, vol. 8, article 10, 2009.

[30] S. Tyrovolas, E. Polychronopoulos, Y. Tountas, and D. B. Panagiotakos, "Modelling nutritional services, in relation to the health status of older individuals living on Mediterranean islands," . under review.

[31] S. Tyrovolas, E. Polychronopoulos, Y. Tountas, and D. B. Panagiotakos, "Evaluating the role of nutritional services in the formation of dietary habits in an elderly population living in the Mediterranean islands," . under review.

[32] L. H. Lindholm, A. D. Koutis, C. D. Lionis, I. G. Vlachonikolis, A. Isacsson, and M. Fioretos, "Risk factors for ischaemic heart disease in a Greek population. A cross-sectional study of men and women living in the village of Spili in Crete," European Heart Journal, vol. 13, no. 3, pp. 291-298, 1992.

[33] C. I. Vardavas, M. K. Linardakis, C. M. Hatzis, W. H.M. Saris, and A. G. Kafatos, "Cardiovascular disease risk factors and dietary habits of farmers from Crete 45 years after the first description of the Mediterranean diet," European Journal of Cardiovascular Prevention and Rehabilitation, vol. 17, no. 4, pp. 440-446, 2010.

[34] C. M. Barbagallo, G. Cavera, M. Sapienza et al., "Prevalence of overweight and obesity in a rural southern Italy population and relationships with total and cardiovascular mortality: the Ventimiglia di Sicilia project," International Journal of Obesity, vol. 25, no. 2, pp. 185-190, 2001. 
[35] C. M. Barbagallo, G. Cavera, M. Sapienza et al., "Nutritional characteristics of a rural Southern Italy population: the Ventimiglia di Sicilia Project," Journal of the American College of Nutrition, vol. 21, no. 6, pp. 523-529, 2002.

[36] J. A. Tur, D. Romaguera, and A. Pons, "Adherence to the Mediterranean dietary pattern among the population of the Balearic Islands," British Journal of Nutrition, vol. 92, no. 3, pp. 341-346, 2004.

[37] J. A. Tur, L. Serra-Majem, D. Romaguera, and A. Pons, "Does the diet of the Balearic population, a Mediterranean type diet, still provide adequate antioxidant nutrient intakes?” European Journal of Nutrition, vol. 44, no. 4, pp. 204-213, 2005.

[38] S. Tessier and M. Gerber, "Factors determining the nutrition transition in two Mediterranean islands: sardinia and Malta," Public Health Nutrition, vol. 8, no. 8, pp. 1286-1292, 2005. 


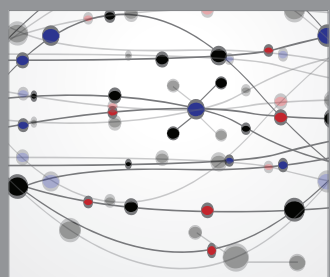

The Scientific World Journal
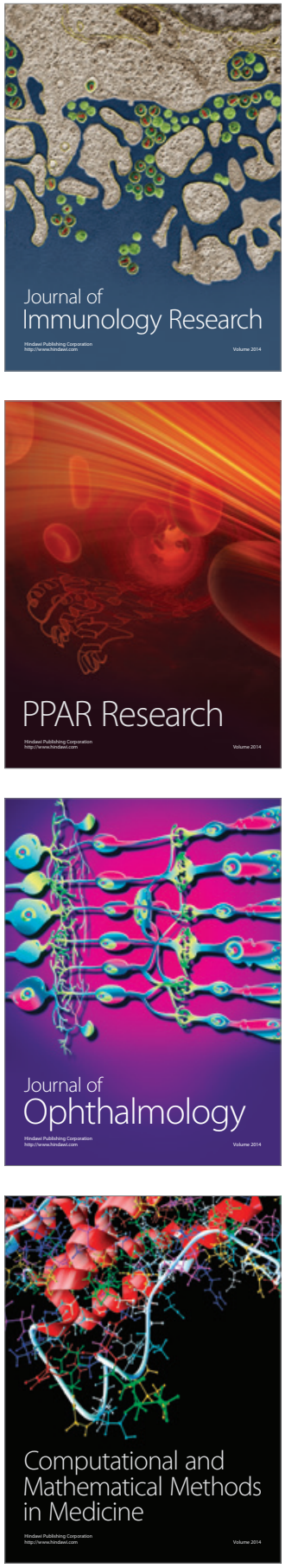

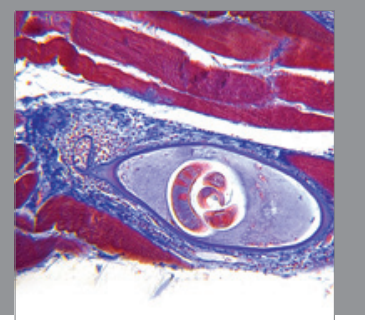

Gastroenterology

Research and Practice
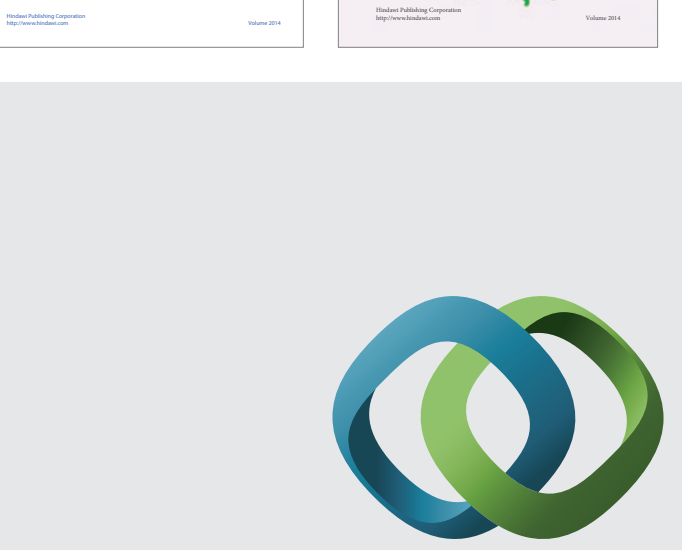

\section{Hindawi}

Submit your manuscripts at

http://www.hindawi.com
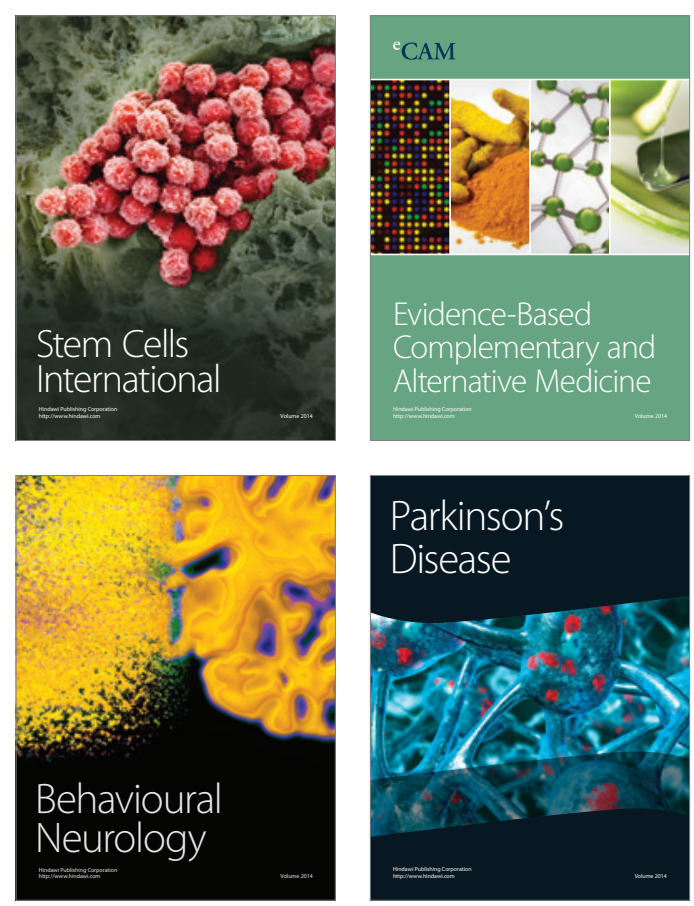

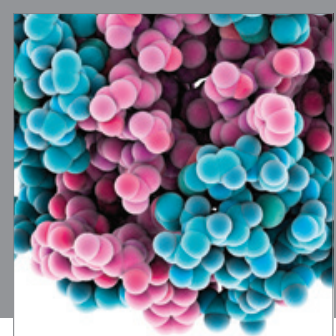

Journal of
Diabetes Research

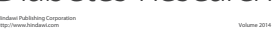

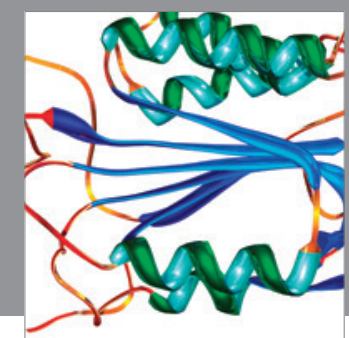

Disease Markers
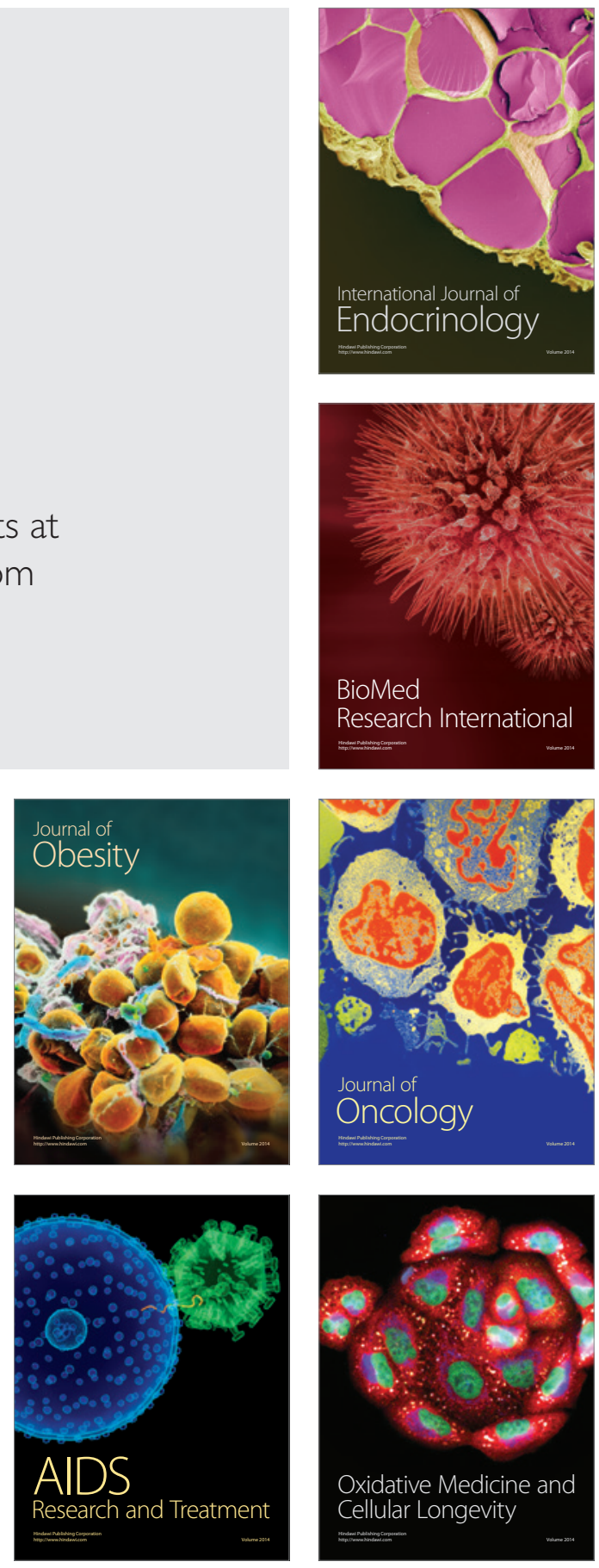